\title{
Global existence of solutions for interval-valued integro-differential equations under generalized $H$-differentiability
}

\author{
Vinh An Truong ${ }^{1}$, Van Hoa Ngo ${ }^{2 *}$ and Dinh Phu Nguyen ${ }^{3}$
}

\author{
"Correspondence: \\ ngovanhoa_clt@yahoo.com \\ 2Division of Applied Mathematics, \\ Ton Duc Thang University, Nguyen \\ Huu Tho Street, District 7, Ho Chi \\ Minh City, Vietnam \\ Full list of author information is \\ available at the end of the article
}

\begin{abstract}
In this study, we consider the interval-valued integro-differential equations (IIDEs) under generalized $H$-differentiability

$$
D_{H}^{g} X(t)=F(t, X(t))+\int_{t_{0}}^{t} G(t, s, X(s)) d s, \quad X\left(t_{0}\right)=X_{0} \in K_{C}(\mathbb{R}) .
$$

The global existence of solutions for interval-valued integro-differential equations with initial conditions under generalized $\mathrm{H}$-differentiability is studied. Theorems for global existence of solutions are given and proved on $\left[t_{0}, \infty\right)$. Some examples are given to illustrate these results.
\end{abstract}

MSC: $34 \mathrm{KO} 05 ; 34 \mathrm{~K} 30 ; 47 \mathrm{G} 20$

Keywords: interval-valued differential equations; interval-valued integro-differential equations; generalized Hukuhara derivative

\section{Introduction}

The set-valued differential and integral equations are an important part of the theory of set-valued analysis, and they play an important role in the theory and application of control theory; and they were first studied in 1969 by De Blasi and Iervolino [1]. Recently, set-valued differential equations have been studied by many scientists due to their applications in many areas. For the basic theory on set-valued differential and integral equations, the readers can be referred to the following books and papers [2-13] and references therein. Integro-differential equations are encountered in many areas of science, where it is necessary to take into account aftereffect or delay (for example, in control theory, biology, ecology, medicine, etc. [14-16]). Especially, one always describes a model which possesses hereditary properties by integro-differential equations in practice.

The interval-valued analysis and interval differential equations (IDEs) are the special cases of the set-valued analysis and set-valued differential equations, respectively. In many situations, when modeling real-world phenomena, information about the behavior of a dynamic system is uncertain and one has to consider these uncertainties to gain better meaning of full models. Interval-valued differential equation is a natural way to model dynamic systems subject to uncertainties. Recently, many works have been done by several authors in the theory of interval-valued differential equations (see, e.g., [17-20]). There are several 
approaches to the study of interval differential equations. One popular approach is based on $H$-differentiability. The approach based on $H$-derivative has the disadvantage that it leads to solutions which have an increasing length of their support. Recently, Stefanini and Bede [17] solved the above mentioned approach under strongly generalized differentiability of interval-valued functions. In this case, the derivative exists and the solution of an interval-valued differential equation may have decreasing length of the support, but the uniqueness is lost. The paper of Stefanini and Bede was the starting point for the topic of interval-valued differential equations (see $[19,20]$ ) and later also for fuzzy differential equations. Also, a very important generalization and development related to the subject of the present paper is in the field of fuzzy sets, i.e., fuzzy calculus and fuzzy differential equations under the generalized Hukuhara derivative. Recently, several works, e.g., [7, 10, 16, 21-39], have been done on set-valued differential equations, fuzzy differential equations and random fuzzy differential equations.

In $[17,19,20]$ the authors presented interval-valued differential equations under generalized Hukuhara differentiability which were given the following form:

$$
X^{\prime}(t)=F(t, X(t)), \quad X\left(t_{0}\right)=X_{0} \in K_{C}(\mathbb{R}), t \in\left[t_{0}, T\right]
$$

where' denotes two kinds of derivatives, namely the classical Hukuhara derivative and the second type Hukuhara derivative (generalized Hukuhara differentiability). The existence and uniqueness of a Cauchy problem is then obtained under an assumption that the coefficients satisfy a condition with the Lipschitz constant (see [17]). The proof is based on the application of the Banach fixed point theorem. In [20], under the generalized Lipschitz condition, Malinowski obtained the existence and uniqueness of solutions to both kinds of IDEs.

In this paper, we study two kinds of solutions to IIDEs. The different types of solutions to IIDEs are generated by the usage of two different concepts of interval-valued derivative. This direction of research is motivated by the results of Stefanini and Bede [17], Malinowski $[19,20]$ concerning deterministic IDEs with generalized interval-valued derivative.

This paper is organized as follows. In Section 2, we recall some basic concepts and notations about interval analysis and interval-valued differential equations. In Section 3, we present the global existence of solutions to the interval-valued integro-differential equations under two kinds of the Hukuhara derivative. Finally, we give some examples for IIDEs in Section 4.

\section{Preliminaries}

Let $K_{C}\left(\mathbb{R}^{n}\right)$ be the space of nonempty compact and convex sets of $\mathbb{R}^{n}$. The set of real intervals will be denoted by $K_{C}(\mathbb{R})$. The addition and scalar multiplication in $K_{C}(\mathbb{R})$, we define as usual, i.e., for $A, B \in K_{C}(\mathbb{R}), A=\left[a^{-}, a^{+}\right], B=\left[b^{-}, b^{+}\right]$, where $a^{-} \leq a^{+}, b^{-} \leq b^{+}$, and $\lambda \geq 0$, then we have

$$
A+B=\left[a^{-}+b^{-}, a^{+}+b^{+}\right], \quad \lambda A=\left[\lambda a^{-}, \lambda a^{+}\right] \quad\left(-\lambda A=\left[\lambda a^{+}, \lambda a^{-}\right]\right) .
$$

Furthermore, let $A \in K_{C}(\mathbb{R}), \lambda_{1}, \lambda_{2}, \lambda_{3}, \lambda_{4} \in \mathbb{R}$ and $\lambda_{3}, \lambda_{4} \geq 0$, then we have $\lambda_{1}\left(\lambda_{2} A\right)=$ $\left(\lambda_{1} \lambda_{2}\right) A$ and $\left(\lambda_{3}+\lambda_{4}\right) A=\lambda_{3} A+\lambda_{4} A$. Let $A, B \in K_{C}(\mathbb{R})$ as above. Then the Hausdorff metric 
$H$ in $K_{C}(\mathbb{R})$ is defined as follows:

$$
H(A, B)=\max \left\{\left|a^{-}-b^{-}\right|,\left|a^{+}-b^{+}\right|\right\} .
$$

We notice that $\left(K_{C}(\mathbb{R}), H\right)$ is a complete, separable and locally compact metric space. We define the magnitude and the length of $A \in K_{C}(\mathbb{R})$ by

$$
H(A,\{0\})=\|A\|=\max \left\{\left|a^{-}\right|,\left|a^{+}\right|\right\}, \quad \operatorname{len}(A)=a^{+}-a^{-}
$$

respectively, where $\{0\}$ is the zero element of $K_{C}(\mathbb{R})$, which is regarded as one point. The Hausdorff metric (2.1) satisfies the following properties:

$$
\begin{aligned}
& H(A+C, B+C)=H(A, B) \text { and } H(A, B)=H(B, A), \\
& H(A+B, C+D) \leq H(A, C)+H(B, D), \\
& H(\lambda A, \lambda B)=|\lambda| H(A, B)
\end{aligned}
$$

for all $A, B, C, D \in K_{C}(\mathbb{R})$ and $\lambda \in \mathbb{R}$. Let $A, B \in K_{C}(\mathbb{R})$. If there exists an interval $C \in K_{C}(\mathbb{R})$ such that $A=B+C$, then we call $C$ the Hukuhara difference of $A$ and $B$. We denote the interval $C$ by $A \ominus B$. Note that $A \ominus B \neq A+(-) B$. It is known that $A \ominus B$ exists in the case len $(A) \geq \operatorname{len}(B)$. Besides that, we can see $[19,20,40,41]$ the following properties for $A, B, C, D \in K_{C}(\mathbb{R})$ :

- If $A \ominus B, A \ominus C$ exist, then $H(A \ominus B, A \ominus C)=H(B, C)$;

- If $A \ominus B, C \ominus D$ exist, then $H(A \ominus B, C \ominus D)=H(A+D, B+C)$;

- If $A \ominus B, A \ominus(B+C)$ exist, then there exist $(A \ominus B) \ominus C$ and $(A \ominus B) \ominus C=A \ominus(B+C)$;

- If $A \ominus B, A \ominus C, C \ominus B$ exist, then there exist $(A \ominus B) \ominus(A \ominus C)$ and $(A \ominus B) \ominus(A \ominus C)=C \ominus B$.

Definition 2.1 [20] We say that the interval-valued mapping $X:[a, b] \subset R^{+} \rightarrow K_{C}(\mathbb{R})$ is continuous at the point $t \in[a, b]$ if for every $\epsilon>0$ there exists $\delta=\delta(t, \epsilon)>0$ such that, for all $s \in[a, b]$ such that $|t-s|<\delta$, one has $H(X(t), X(s)) \leq \epsilon$.

The strongly generalized differentiability was introduced in [17] and studied in [19, $36-38]$.

Definition 2.2 Let $X:[a, b] \rightarrow K_{C}(\mathbb{R})$ and $t \in[a, b]$. We say that $X$ is strongly generalized differentiable at $t$ if there exists $D_{H}^{g} X(t) \in K_{C}(\mathbb{R})$ such that

(i) for all $h>0$ sufficiently small, $\exists X(t+h) \ominus X(t), \exists X(t) \ominus X(t-h)$ and the limits

$$
\lim _{h \searrow 0} H\left(\frac{X(t+h) \ominus X(t)}{h}, D_{H}^{g} X(t)\right)=0, \quad \lim _{h \searrow 0} H\left(\frac{X(t) \ominus X(t-h)}{h}, D_{H}^{g} X(t)\right)=0 \text {, }
$$

or

(ii) for all $h>0$ sufficiently small, $\exists X(t) \ominus X(t+h), \exists X(t-h) \ominus X(t)$ and the limits

$$
\lim _{h \searrow 0} H\left(\frac{X(t) \ominus X(t+h)}{-h}, D_{H}^{g} X(t)\right)=0, \quad \lim _{h \searrow 0} H\left(\frac{X(t-h) \ominus X(t)}{-h}, D_{H}^{g} X(t)\right)=0,
$$


(iii) for all $h>0$ sufficiently small, $\exists X(t+h) \ominus X(t), \exists X(t-h) \ominus X(t)$ and the limits

$$
\lim _{h \searrow 0} H\left(\frac{X(t+h) \ominus X(t)}{h}, D_{H}^{g} X(t)\right)=0, \quad \lim _{h \searrow 0} H\left(\frac{X(t-h) \ominus X(t)}{h}, D_{H}^{g} X(t)\right)=0,
$$

or

(iv) for all $h>0$ sufficiently small, $\exists X(t) \ominus X(t+h), \exists X(t) \ominus X(t-h)$ and the limits

$$
\lim _{h \searrow 0} H\left(\frac{X(t) \ominus X(t+h)}{-h}, D_{H}^{g} X(t)\right)=0, \quad \lim _{h \searrow 0} H\left(\frac{X(t) \ominus X(t-h)}{-h}, D_{H}^{g} X(t)\right)=0
$$

( $h$ at denominators means $\frac{1}{h}$ ). In this definition, case (i) ((i)-differentiability for short) corresponds to the classical $H$-derivative, so this differentiability concept is a generalization of the Hukuhara derivative. In [17], Stefanini and Bede considered four cases for the derivative. In this paper, we consider only the two first items of Definition 2.2. In other cases, the derivative is trivial because it is reduced to a crisp element.

Remark 2.1 $[19,41]$ If for intervals $X, Y, Z \in K_{C}(\mathbb{R})$ there exist Hukuhara differences $X \ominus$ $Y, X \ominus Z$, then $H(X \ominus Y,\{0\})=H(X, Y)$ and $H(X \ominus Y, X \ominus Z)=H(Y, Z)$.

Let $X, Y:[a, b] \rightarrow K_{C}(\mathbb{R})$. We have (see [8]) some properties of $D_{H}^{g}$ as follows:

(i) If $X$ is (i)-differentiable, then it is continuous.

(ii) If $X, Y$ are (i)-differentiable and $\lambda \in \mathbb{R}$, then $D_{H}^{g}(X+Y)(t)=D_{H}^{g} X(t)+D_{H}^{g} Y(t)$, $D_{H}^{g}(\lambda X)(t)=\lambda D_{H}^{g} X(t)$.

(iii) Let $X$ be (i)-differentiable and assume that $D_{H}^{g} X$ is integrable over $[a, b]$. Then we have $X(t)=X(a)+\int_{a}^{t} D_{H}^{g} X(s) d s$.

(iv) If $X$ is (i)-differentiable on $[a, b]$, then the real function $t \rightarrow \operatorname{len}(X(t))$ is nondecreasing on $[a, b]$.

(v) Let $X$ be (ii)-differentiable and assume that $D_{H}^{g} X$ is integrable over $[a, b]$. Then we have $X(a)=X(t)+(-1) \int_{a}^{t} D_{H}^{g} X(s) d s$.

(vi) If $X$ is (ii)-differentiable on $[a, b]$, then the real function $t \rightarrow \operatorname{len}(X(t))$ is nonincreasing on $[a, b]$.

Corollary 2.1 (see, e.g., $[17,19]$ ) Let $X:\left[t_{0}, T\right] \rightarrow K_{C}(\mathbb{R})$ be given. Denote $X(t)=\left[X^{-}(t)\right.$, $\left.X^{+}(t)\right]$ for $t \in\left[t_{0}, T\right]$, where $X^{-}, X^{+}:\left[t_{0}, T\right] \rightarrow \mathbb{R}$.

(i) If the mapping $X$ is (i)-differentiable (i.e., classical Hukuhara differentiability) at $t \in\left[t_{0}, T\right]$, then the real-valued functions $X^{-}, X^{+}$are differentiable at $t$ and $D_{H}^{g} X(t)=\left[\left(X^{-}\right)^{\prime}(t),\left(X^{+}\right)^{\prime}(t)\right]$.

(ii) If the mapping $X$ is (ii)-differentiable at $t \in\left[t_{0}, T\right]$, then the real-valued functions $X^{-}$, $X^{+}$are differentiable at $t$ and $D_{H}^{g} X(t)=\left[\left(X^{+}\right)^{\prime}(t),\left(X^{-}\right)^{\prime}(t)\right]$.

Lemma 2.1 (see $[17,19,20])$ The interval-valued differential equation $D_{H}^{g} X(t)=F(t, X(t))$, $X\left(t_{0}\right)=X_{0} \in K_{C}(\mathbb{R})$, where $F:\left[t_{0}, T\right] \times K_{C}(\mathbb{R}) \rightarrow K_{C}(\mathbb{R})$ is supposed to be continuous, is equivalent to one of the integral equations

$$
X(t)=X_{0}+\int_{t_{0}}^{t} F(s, X(s)) d s, \quad \forall t \in\left[t_{0}, T\right]
$$


or

$$
X_{0}=X(t)+(-1) \int_{a}^{t} F(s, X(s)) d s, \quad \forall t \in\left[t_{0}, T\right]
$$

on the interval $\left[t_{0}, T\right] \in \mathbb{R}$, under the strong differentiability condition, (i) or (ii), respectively. We notice that the equivalence between two equations in this lemma means that any solution is a solution for the other one.

We consider the Cauchy problem for IIDEs under the form

$$
D_{H}^{g} X(t)=F(t, X(t))+\int_{t_{0}}^{t} K(t, s, X(s)) d s, \quad X\left(t_{0}\right)=X_{0}
$$

for all $t \in\left[t_{0}, T\right]$, where $F: I=\left[t_{0}, T\right] \times K_{C}(\mathbb{R}) \rightarrow K_{C}(\mathbb{R})$ and $K: \mathcal{D} \times K_{C}(\mathbb{R}) \rightarrow K_{C}(\mathbb{R})$ are continuous interval-valued mappings on $I$, with $\mathcal{D}=\left\{(t, s) \in I \times I: t_{0} \leq s \leq t<T\right\}$.

Definition 2.3 A mapping $X:\left[t_{0}, T\right] \rightarrow K_{C}(\mathbb{R})$ is called a solution to problem (2.2) on $I$ if and only if $X$ is a continuous mapping on $I$ and it satisfies one of the following intervalvalued integral equations:

(S1) $X(t)=X_{0}+\left(\int_{t_{0}}^{t} F(s, X(s)) d s+\int_{t_{0}}^{t} \int_{t_{0}}^{s} K(s, u, X(u)) d u d s\right), t \in I$, if $X$ is (i)-differentiable or (iii)-differentiable.

(S2) $X(t)=X_{0} \ominus(-1)\left(\int_{t_{0}}^{t} F(s, X(s)) d s+\int_{t_{0}}^{t} \int_{t_{0}}^{s} K(s, u, X(u)) d u d s\right), t \in I$, if $X$ is (ii)-differentiable or (iv)-differentiable.

Definition 2.4 Let $X:\left[t_{0}, T\right] \rightarrow K_{C}(\mathbb{R})$ be an interval-valued function which is (i)differentiable. If $X$ and its derivative satisfy problem (2.2), we say $X$ is a (i)-solution of problem (2.2).

Definition 2.5 Let $X:\left[t_{0}, T\right] \rightarrow K_{C}(\mathbb{R})$ be an interval-valued function which is (ii)differentiable. If $X$ and its derivative satisfy problem (2.2), we say $X$ is a (ii)-solution of problem (2.2).

Definition 2.6 A solution $X:\left[t_{0}, T\right] \rightarrow K_{C}(\mathbb{R})$ is unique if $\sup _{t \in\left[t_{0}, T\right]} H(X(t), Y(t))=0$ for any mapping $Y:\left[t_{0}, T\right] \rightarrow K_{C}(\mathbb{R})$ that is a solution to $(2.2)$ on $\left[t_{0}, T\right]$.

Theorem 2.1 (see $[42]$ ) Let $F: I=\left[t_{0}, T\right] \times K_{C}(\mathbb{R}) \rightarrow K_{C}(\mathbb{R})$ and $K: \mathcal{D} \times K_{C}(\mathbb{R}) \rightarrow K_{C}(\mathbb{R})$ be continuous interval-valued mappings on $I$. Suppose that there exists $L>0$ such that

$$
\max \left\{H\left(F\left(t, X_{1}\right), F\left(t, X_{2}\right)\right), H\left(K\left(t, s, X_{1}\right), K\left(t, s, X_{2}\right)\right)\right\} \leq L H\left(X_{1}, X_{2}\right)
$$

for all $t, s \in I, X_{1}, X_{2} \in K_{C}(\mathbb{R})$. Then there exists the only local solution $X$ to IIDE (2.2) on some intervals $\left[t_{0}, \mathbb{T}\right]\left(\mathbb{T} \leq T-t_{0}\right)$ for each case ((i)-solution and (ii)-solution).

\section{Main results}

In this section of the paper, we consider again the following initial value problem for the interval-valued integro-differential equations (IIDEs) under the form

$$
D_{H}^{g} X(t)=F(t, X(t))+\int_{t_{0}}^{t} K(t, s, X(s)) d s, \quad X\left(t_{0}\right)=X_{0}
$$


for all $t \in J=\left[t_{0}, \infty\right)$, where $F: J \times K_{C}(\mathbb{R}) \rightarrow K_{C}(\mathbb{R})$ and $K: \mathcal{D} \times K_{C}(\mathbb{R}) \rightarrow K_{C}(\mathbb{R})$ are continuous interval-valued mappings on $J$, with $\mathcal{D}=\left\{(t, s) \in J \times J: t_{0} \leq s \leq t<\infty\right\}$.

Theorem 3.1 Assume that

(i) $F(t, X), G(t, s, X)$ are locally Lipschitzian for all $t, s \in J, X \in K_{C}(\mathbb{R})$;

(ii) $f \in C U \times[0, \infty),[0, \infty)]$ and $k \in C[\mathcal{D} \times[0, \infty),[0, \infty)]$ are nondecreasing in $x \geq 0$, and the maximal solution $r\left(t, t_{0}, x_{0}\right)$ of the scalar integro-differential equation

$$
x^{\prime}(t)=f(t, x(t))+\int_{t_{0}}^{t} k(t, s, x(s)) d s, \quad x\left(t_{0}\right)=x_{0} \geq 0,
$$

exists throughout $J$;

(iii) $H(F(t, X),\{0\}) \leq f(t, H(X,\{0\})), H(K(t, s, X),\{0\}) \leq k(t, s, H(X,\{0\}))$ for all $t, s \in J$, $X \in K_{C}(\mathbb{R})$;

(iv) $H\left(X\left(t, t_{0}, X_{0}\right),\{0\}\right) \leq r\left(t, t_{0}, x_{0}\right), H\left(X_{0},\{0\}\right) \leq x_{0}$.

Then the largest interval of the existence of any solution $X\left(t, t_{0}, X_{0}\right)$ of (3.1) for each case ((i)-solution and (ii)-solution) such that $H\left(X_{0},\{0\}\right) \leq x_{0}$ is $J$. In addition, if $r\left(t, t_{0}, x_{0}\right)$ is bounded on J, then $\lim _{t \rightarrow \infty} X\left(t, t_{0}, X_{0}\right)$ exists in $\left(K_{C}(\mathbb{R}), H\right)$.

Proof Since the way of the proof is similar for both cases ((i)-solution and (ii)-solution), we only prove case (i)-differentiability. By hypothesis (i), there exists a $T>t_{0}$ such that the unique (i)-solution of problem (3.1) exists on $\left[t_{0}, T\right]$. Let

$$
\mathcal{S}=\left\{X(t) \mid X(t) \text { is defined on }\left[t_{0}, \alpha_{X}\right] \text { and is the (i)-solution to (3.1) }\right\} \text {. }
$$

Then $\mathcal{S} \neq \emptyset$. Taking $\alpha=\sup \left\{\alpha_{X} \mid X(t) \in \mathcal{S}\right\}$, clearly, there exists a unique (i)-solution of problem (3.1) which is defined on $\left[t_{0}, \alpha\right)$ with $H\left(X_{0},\{0\}\right) \leq x_{0}$. Next, we shall prove that $\alpha=\infty$. We suppose $\alpha<\infty$ and define

$$
m(t)=H\left(X\left(t, t_{0}, X_{0},\{0\}\right)\right), \quad t_{0} \leq t<\alpha .
$$

Using assumptions (ii) and (iii), we have

$$
\begin{aligned}
D_{+} m(t) & =\liminf _{t \rightarrow+0} \frac{H\left(X\left(t+h, t_{0}, X_{0}\right),\{0\}\right)-H\left(X\left(t, t_{0}, X_{0}\right),\{0\}\right)}{h} \\
& \leq \liminf _{h \rightarrow+0} \frac{H\left(X\left(t+h, t_{0}, X_{0}\right), X\left(t, t_{0}, X_{0}\right)\right)}{h} \\
& =\liminf _{h \rightarrow+0} \frac{H\left(X\left(t+h, t_{0}, X_{0}\right) \ominus X\left(t, t_{0}, X_{0}\right),\{0\}\right)}{h} \\
& =H\left(D_{H}^{g} X(t),\{0\}\right)=H\left(F(t, X(t))+\int_{t_{0}}^{t} K(t, s, X(s)) d s,\{0\}\right) \\
& \leq H(F(t, X(t)),\{0\})+\int_{t_{0}}^{t} H(K(t, s, X(s)),\{0\}) d s \\
& \leq f(t, m(t))+\int_{t_{0}}^{t} k(t, s, m(s)) d s, \quad t_{0} \leq t<\alpha,
\end{aligned}
$$


and $m\left(t_{0}\right)=H\left(X_{0},\{0\}\right) \leq x_{0}$. Further, by assumption (iv), it follows that $m(t) \leq r\left(t, t_{0}, x_{0}\right)$, $t_{0} \leq t<\alpha$. Next, we deduce that $\lim _{t \rightarrow \alpha-0} X\left(t, t_{0}, X_{0}\right)$ exists in $\left(K_{C}(\mathbb{R}), H\right)$. In fact, for any $t_{1}, t_{2}$ such that $t_{0} \leq t_{1}<t_{2}<\alpha$, we obtain

$$
\begin{aligned}
H\left(X\left(t_{1}, t_{0}, X_{0}\right), X\left(t_{2}, t_{0}, X_{0}\right)\right) & \\
= & H\left(\begin{array}{l}
X_{0}+\int_{t_{0}}^{t_{1}} F(s, X(s)) d s+\int_{t_{0}}^{t_{1}} \int_{t_{0}}^{s} K(s, u, X(u)) d u d s, \\
X_{0}+\int_{t_{0}}^{t_{2}} F(s, X(s)) d s+\int_{t_{0}}^{t_{2}} \int_{t_{0}}^{s} K(s, u, X(u)) d u d s
\end{array}\right) \\
\leq & H\left(\int_{t_{0}}^{t_{1}} F(s, X(s)) d s+\int_{t_{0}}^{t_{1}} \int_{t_{0}}^{s} K(s, u, X(u)) d u d s,\right. \\
& \left.\int_{t_{0}}^{t_{2}} F(s, X(s)) d s+\int_{t_{0}}^{t_{2}} \int_{t_{0}}^{s} K(s, u, X(u)) d u d s\right) \\
\leq & \int_{t_{1}}^{t_{2}} H(F(s, X(s)),\{0\}) d s+\int_{t_{1}}^{t_{2}} \int_{t_{0}}^{s} H(K(s, u, X(u)),\{0\}) d u d s \\
\leq & \int_{t_{1}}^{t_{2}} f(s, r(s)) d s+\int_{t_{1}}^{t_{2}} \int_{t_{0}}^{s} k(s, u, r(u)) d u d s=\int_{t_{1}}^{t_{2}} r^{\prime}(s) d s=r\left(t_{2}\right)-r\left(t_{1}\right) .
\end{aligned}
$$

Since $\lim _{t \rightarrow \alpha-0} r\left(t, t_{0}, x_{0}\right)$ exists and is finite, taking the limits as $t_{1}, t_{2} \rightarrow \alpha-0$ and using the completeness of $\left(K_{C}(\mathbb{R}), H\right)$, it follows from the estimate $H\left(X\left(t_{1}, t_{0}, X_{0}\right), X\left(t_{2}, t_{0}, X_{0}\right)\right) \leq$ $r\left(t_{2}\right)-r\left(t_{1}\right)$ that $\lim _{t \rightarrow \alpha-0} X\left(t, t_{0}, X_{0}\right)$ exists in $\left(K_{C}(\mathbb{R}), H\right)$. Now we define $X(\alpha)=$ $\lim _{t \rightarrow \alpha-0} X(t)$ and consider the IVP

$$
D_{H}^{g} X(t)=F(t, X(t))+\int_{t_{0}}^{t} K(t, s, X(s)) d s, \quad X(\alpha)=\lim _{t \rightarrow \alpha-0} X(t) .
$$

By the assumption (i) again, it follows that $X(t)$ can be extended beyond $\alpha$, which contradicts our assumption. So, any (i)-solution of problem (3.1) exists on $J=\left[t_{0}, \infty\right)$, and so $\alpha=\infty$.

Example 3.2 Consider the IIDE

$$
D_{H}^{g} X(t)=a(t) X(t)+\int_{t_{0}}^{t} b(s) X(s) d s, \quad X\left(t_{0}\right)=X_{0},
$$

where we assume that $a(t), b(t): \mathbb{R}^{+} \rightarrow \mathbb{R}^{+}$are continuous functions. We see that $F(t, X(t))=a(t) X(t)$ and $K(t, s, X(t))=b(t) X(t)$ are locally Lipschitzian. If we let $f(t, x(t))=$ $a(t) x(t)$ and $k(t, s, x(t))=b(t) x(t)$, then $x(t) \equiv 0$ is an unique solution of

$$
x^{\prime}(t)=a(t) x(t)+\int_{t_{0}}^{t} b(s) x(s) d s, \quad x\left(t_{0}\right)=0
$$

on $\left[t_{0}, \infty\right)$. Moreover, we see that $H(a(t) X(t),\{0\}) \leq a(t) H(X(t),\{0\})=f(t, H(X(t),\{0\}))$ and $H(b(t) X(t),\{0\}) \leq b(t) H(X(t),\{0\})=k(t, s, H(X(t),\{0\}))$. Therefore, the solutions of problem (3.4) are on $\left[t_{0}, \infty\right)$.

Employing the comparison Theorem 3.1, we shall prove the following global existence result. 


\section{Theorem 3.3 Assume that}

(i) $F \in C\left[J \times K_{C}(\mathbb{R}), K_{C}(\mathbb{R})\right], K \in C\left[\mathcal{D} \times K_{C}(\mathbb{R}), K_{C}(\mathbb{R})\right], F$ and $K$ are bounded on bounded sets, and there exists a local (i)-solution of (3.1) for every $\left(t_{0}, X_{0}\right), t_{0} \geq 0$ and $X_{0} \in K_{C}(\mathbb{R})$;

(ii) $\left.V \in C U \times K_{C}(\mathbb{R}),[0, \infty)\right] ;|V(t, A)-V(t, B)| \leq L H(A, B)$, where $L$ is the local Lipschitz constant, for $A, B \in K_{C}(\mathbb{R}), t \in J, V(t, A) \rightarrow \infty$ as $H(A,\{0\}) \rightarrow \infty$ uniformly for $\left[t_{0}, T\right]$, for every $T>t_{0}$ and for $t \in J, A \in K_{C}(\mathbb{R})$,

$$
\begin{aligned}
& \limsup _{h \rightarrow 0^{+}} \frac{1}{h}\left[V\left(t+h, A+h\left\{F(t, A)+\int_{t_{0}}^{t} K(t, s, A) d s\right\}\right)-V(t, A)\right] \\
& \quad \leq f(t, V(t, A))+\int_{t_{0}}^{t} k(t, s, V(s, A)) d s,
\end{aligned}
$$

where $f \in C U \times[0, \infty), \mathbb{R}], k \in C[\mathcal{D} \times[0, \infty), \mathbb{R}]$;

(iii) The maximal solution $r(t)=r\left(t, t_{0}, x_{0}\right)$ of the scalar integro-differential equation

$$
x^{\prime}(t)=f(t, x(t))+\int_{t_{0}}^{t} k(t, s, x(s)) d s, \quad x\left(t_{0}\right)=x_{0} \geq 0
$$

exists on $J$ and is positive whenever $x_{0}>0$.

Then, for every $X_{0} \in K_{C}(\mathbb{R})$ such that $V\left(t_{0}, X_{0}\right) \leq x_{0}$, problem (3.1) has a (i)-solution $X(t)$ on $\left[t_{0}, \infty\right)$, which satisfies the estimate

$$
V(t, X(t)) \leq r\left(t, t_{0}, x_{0}\right), \quad t \geq t_{0}
$$

Proof Let $\mathbb{S}$ denote the set of all functions $X$ defined on $J_{X}=\left[t_{0}, c_{X}\right)$ with values in $K_{C}(\mathbb{R})$ such that $X(t)$ is a (i)-solution of problem (3.1) on $J_{X}$ and $V(t, X(t)) \leq r(t), t \in J_{X}$. We define a partial order $\leq$ on $\mathbb{S}$ as follows: the relation $X \leq Y$ implies that $J_{X} \subseteq J_{Y}$ and $Y(t) \equiv X(t)$ on $J_{X}$. We shall first show that $\mathbb{S}$ is nonempty. Indeed, by assumption (i), there exists a (i)-solution $X(t)$ of problem (3.1) defined on $J_{X}=\left[t_{0}, c_{X}\right)$. Let $X(t)=X\left(t, t_{0}, X_{0}\right)$ be any (i)solution of (3.1) existing on $J_{X}$. Define $k(t)=V(t, X(t))$ so that $k\left(t_{0}\right)=V\left(t_{0}, X_{0}\right) \leq x_{0}$. Now, for small $h>0$ and using assumption (ii), we consider

$$
\begin{aligned}
k(t+h)-k(t) & \\
= & V(t+h, X(t+h))-V(t, X(t)) \\
\leq & V(t+h, X(t+h))+V\left(t+h, X(t)+h\left\{F(t, X(t))+\int_{t_{0}}^{t} K(t, s, X(s)) d s\right\}\right) \\
& -V\left(t+h, X(t)+h\left\{F(t, X(t))+\int_{t_{0}}^{t} K(t, s, X(s)) d s\right\}\right)-V(t, X(t)) \\
\leq & L H\left(X(t+h), X(t)+h\left\{F\left(t, X(t)+\int_{t_{0}}^{t} K(t, s, X(s)) d s\right)\right\}\right) \\
& +V\left(t+h, X(t)+h\left\{F(t, X(t))+\int_{t_{0}}^{t} K(t, s, X(s)) d s\right\}\right)-V(t, X(t))
\end{aligned}
$$


using the Lipschitz condition in assumption (ii). Thus

$$
\begin{aligned}
D^{+} k(t) \equiv \limsup _{h \rightarrow 0^{+}} \frac{1}{h}[k(t+h)-k(t)] \\
\leq D^{+} V(t, X(t)) \\
\quad+L \limsup _{h \rightarrow 0^{+}} \frac{1}{h} H\left(X(t+h), X(t)+h\left\{F(t, X(t))+\int_{t_{0}}^{t} K(t, s, X(s)) d s\right\}\right) .
\end{aligned}
$$

Since

$$
\begin{array}{r}
\frac{1}{h} H\left(X(t+h), X(t)+h\left\{F(t, X(t))+\int_{t_{0}}^{t} K(t, s, X(s)) d s\right\}\right) \\
=H\left(\frac{X(t+h) \ominus X(t)}{h}, F(t, X(t))+\int_{t_{0}}^{t} K(t, s, X(s)) d s\right)
\end{array}
$$

and $X(t)$ is any (i)-solution of (3.1), we find that

$$
\begin{gathered}
\limsup _{h \rightarrow 0^{+}} \frac{1}{h} H\left(X(t+h), X(t)+h\left\{F(t, X(t))+\int_{t_{0}}^{t} K(t, s, X(s)) d s\right\}\right) \\
\quad=\limsup _{h \rightarrow 0^{+}} H\left(\frac{X(t+h) \ominus X(t)}{h}, F(t, X(t))+\int_{t_{0}}^{t} K(t, s, X(s)) d s\right) \\
=H\left[D_{H}^{g} X(t), F(t, X(t))+\int_{t_{0}}^{t} K(t, s, X(s)) d s\right]=0 .
\end{gathered}
$$

Therefore, we have the scalar integro-differential inequality

$$
D^{+} k(t) \leq f(t, k(t))+\int_{t_{0}}^{t} k(t, s, x(s)) d s, \quad k\left(t_{0}\right) \leq x_{0} .
$$

According to Proposition 2.3 in [12], we get the estimate

$$
k(t) \leq r\left(t, t_{0}, x_{0}\right), \quad t \in I_{X} .
$$

It follows that

$$
V(t, X(t)) \leq r\left(t, t_{0}, x_{0}\right), \quad t \in I_{X}
$$

where $r(t)$ is the maximal solution of (3.5). This shows that $X \in \mathbb{S}$, and so $\mathbb{S}$ is nonempty. If $\left(X_{\beta}\right)_{\beta}$ is a chain $(\mathbb{S}, \leq)$, then there is a uniquely defined mapping $Y$ on $J_{Y}=\left[t_{0}, \sup _{\beta} c_{X_{\beta}}\right]$ that coincides with $X_{\beta}$ on $J_{X_{\beta}}$. Clearly, $Y \in \mathbb{S}$ and therefore $Y$ is an upper bound of $\left(X_{\beta}\right)_{\beta}$ in $(\mathbb{S}, \leq)$. The proof of the theorem is completed if we show that $c_{Z}=\infty$. Suppose that it is not true, so that $c_{Z}<\infty$. Since $r(t)$ is assumed to exist on $\left[t_{0}, \infty\right), r(t)$ is bounded on $J_{Z}$. Since $V(t, X(t)) \rightarrow \infty$ as $H(X(t),\{0\}) \rightarrow \infty$ uniformly in $t$ on $\left[t_{0}, c_{Z}\right]$, the relation $V(t, X(t)) \leq r(t)$ on $J_{Z}$ implies that $H(Z(t),\{0\})$ is bounded on $J_{Z}$. By assumption (i), this shows that there is an $M>0$ such that

$$
\max \{H(F(t, X(t)),\{0\}), H(K(t, s, X(t)),\{0\})\} \leq M, \quad t \in J_{Z} .
$$


We have, for all $t_{1}, t_{2} \in J_{Z}$ with $t_{1} \leq t_{2}$,

$$
\begin{aligned}
H\left(Z\left(t_{2}\right), Z\left(t_{1}\right)\right) & =H\left(\begin{array}{c}
X_{0}+\left(\int_{t_{0}}^{t_{2}} F(s, Z(s)) d s+\int_{t_{0}}^{t_{2}} \int_{t_{0}}^{s} K(s, u, Z(u)) d u d s\right), \\
X_{0}+\left(\int_{t_{0}}^{t_{1}} F(s, Z(s)) d s+\int_{t_{0}}^{t_{1}} \int_{t_{0}}^{s} K(s, u, Z(u)) d u d s\right)
\end{array}\right) \\
& \leq \int_{t_{1}}^{t_{2}} H(F(s, Z(s)),\{0\}) d s+\int_{t_{1}}^{t_{2}} \int_{t_{0}}^{s} H(K(s, u, Z(u)),\{0\}) d u d s \\
& \leq 2 M\left(t_{2}-t_{1}\right),
\end{aligned}
$$

where $t_{0} \leq t_{1}<t_{2}$. Hence, $Z$ is Lipschitzian on $J_{Z}$ and consequently has a continuous extension $Z_{0}$ on $\left[t_{0}, c_{Z}\right]$. By continuity of $Z_{0}$, we get

$$
Z_{0}\left(c_{Z}\right)=X_{0}+\left(\int_{t_{0}}^{c_{Z}} F(s, Z(s)) d s+\int_{t_{0}}^{c_{Z}} \int_{t_{0}}^{s} K\left(s, u, Z_{0}(u)\right) d u d s\right)
$$

This implies that $Z_{0}(t)$ is a (i)-solution of problem (3.1) on $\left[t_{0}, c_{Z}\right]$ and, clearly, $V\left(t, Z_{0}(t)\right)<$ $r(t), t \in\left[t_{0}, c_{Z}\right]$. Consider the problem

$$
D_{H}^{g} X(t)=F(t, X(t))+\int_{t_{0}}^{t} K(t, s, X(s)) d s, \quad X\left(t_{0}\right)=Z_{0}\left(c_{Z}\right) .
$$

The assumption of local existence implies that there exists a (i)-solution $X_{0}(t)$ on $\left[c_{Z}, c_{Z}+\right.$ $\delta), \delta>0$. Define

$$
Z_{1}(t)= \begin{cases}Z_{0}(t) & \text { for } t \in\left[t_{0}, c_{Z}\right] \\ X_{0}(t) & \text { for } t \in\left[c_{Z}, c_{Z}+\delta\right]\end{cases}
$$

Therefore $Z_{1}(t)$ is a (i)-solution of problem (3.1) on $\left[t_{0}, c_{Z}+\delta\right)$, and, by repeating the arguments that were used to obtain (3.7), we get $V\left(t, Z_{1}(t)\right) \leq r(t), t \in\left[t_{0}, c_{Z}+\delta\right)$. This contradicts the maximality of $Z$, and hence $c_{Z}=+\infty$. The proof is complete.

\section{Corollary 3.1 Assume that}

(i) $F \in C\left[\times K_{C}(\mathbb{R}), K_{C}(\mathbb{R})\right], K \in C\left[\mathcal{D} \times K_{C}(\mathbb{R}), K_{C}(\mathbb{R})\right], F$ and $K$ are bounded on bounded sets, and there exists a local (ii)-solution of (3.1) for every $\left(t_{0}, X_{0}\right), t_{0} \geq 0$ and $X_{0} \in K_{C}(\mathbb{R})$;

(ii) $V \in C\left[\times K_{C}(\mathbb{R}),[0, \infty)\right] ;|V(t, A)-V(t, B)| \leq L H(A, B)$, where $L$ is the local Lipschitz constant, for $A, B \in K_{C}(\mathbb{R}), t \in J, V(t, A) \rightarrow \infty$ as $H(A,\{0\}) \rightarrow \infty$ uniformly for $\left[t_{0}, T\right]$, for every $T>t_{0}$ and for $t \in J, A \in K_{C}(\mathbb{R})$,

$$
\begin{aligned}
& \limsup _{h \rightarrow 0^{+}} \frac{1}{h}\left[V\left(t+h, A \ominus(-1) h\left\{F(t, A)+\int_{t_{0}}^{t} K(t, s, A) d s\right\}\right)-V(t, A)\right] \\
& \quad \leq f(t, V(t, A))+\int_{t_{0}}^{t} k(t, s, V(s, A)) d s,
\end{aligned}
$$

where $f \in C U \times[0, \infty), \mathbb{R}], k \in C[\mathcal{D} \times[0, \infty), \mathbb{R}]$; 
(iii) The maximal solution $r(t)=r\left(t, t_{0}, x_{0}\right)$ of the scalar integro-differential equation

$$
x^{\prime}(t)=f(t, x(t))+\int_{t_{0}}^{t} k(t, s, x(s)) d s, \quad x\left(t_{0}\right)=x_{0} \geq 0,
$$

exists on $J$ and is positive whenever $x_{0}>0$.

Then, for every $X_{0} \in K_{C}(\mathbb{R})$ such that $V\left(t_{0}, X_{0}\right) \leq x_{0}$, problem (3.1) has a (ii)-solution $X(t)$ on $\left[t_{0}, \infty\right)$, which satisfies the estimate

$$
V(t, X(t)) \leq r\left(t, t_{0}, x_{0}\right), \quad t \geq t_{0}
$$

Proof One can obtain this result easily by using the methods as in the proof of Theorem 3.3.

\section{Some examples}

In this section, we present some examples being simple illustrations of the theory of IIDEs. We will consider IIDEs (3.1) with (i) and (ii) derivatives, respectively. For convenience, from now on, we denote the solution of IIDE (3.1) with (i) derivative by $X_{1}$ and the solution with (ii) derivative by $X_{2}$.

Let us start the illustrations by considering the following interval-valued integrodifferential equation:

$$
D_{H}^{g} X(t)=F(t)+\int_{t_{0}}^{t} k(t, s) X(s) d s, \quad X\left(t_{0}\right)=X_{0}=\left[X_{0}^{-}, X_{0}^{+}\right], t \in\left[t_{0}, T\right]
$$

where $F:\left[t_{0}, T\right] \rightarrow K_{C}(\mathbb{R})$ is an interval-valued function $\left(i . e ., F(t)=\left[F^{-}(t), F^{+}(t)\right]\right), k(t, s)$ is a real known function, and $X_{0} \in K_{C}(\mathbb{R})$. In equation (4.1), we shall solve it by two types of the Hukuhara derivative, which are defined in Definition 2.2. Consequently, based on the type of differentiability, we have the following two cases.

Case 1: Suppose that $X(t)$ in equation (4.1) is (i)-differentiable. By using Corollary 2.1, then we get $D_{H}^{g} X(t)=\left[\left(X^{-}(t)\right)^{\prime},\left(X^{+}(t)\right)^{\prime}\right]$. Hence, we have the following:

$$
\left\{\begin{array}{l}
\left(X^{-}(t)\right)^{\prime}=F^{-}(t)+\int_{t_{0}}^{t} \frac{k(t, s) X(s) d s}{\overline{k(t, s) X(s) d s}} \\
\left(X^{+}(t)\right)^{\prime}=F^{+}(t)+\int_{t_{0}}^{t}
\end{array}\right.
$$

where

$$
\underline{k(t, s) X(s) d s}= \begin{cases}k(t, s) X^{-}(t), & k(t, s) \geq 0 \\ k(t, s) X^{+}(t), & k(t, s)<0\end{cases}
$$

and

$$
\overline{k(t, s) X(s) d s}= \begin{cases}k(t, s) X^{+}(t), & k(t, s) \geq 0 \\ k(t, s) X^{-}(t), & k(t, s)<0\end{cases}
$$


From (4.2), we have

$$
\left\{\begin{array}{l}
\left(X^{-}(t)\right)^{\prime}=F^{-}(t)+\int_{t_{0}}^{t} \overline{k(t, s) X(s)} d s \\
\left(X^{+}(t)\right)^{\prime}=F^{+}(t)+\int_{t_{0}}^{t} \overline{k(t, s) X(s)} d s \\
X^{-}\left(t_{0}\right)=X_{0}^{-} \\
X^{+}\left(t_{0}\right)=X_{0}^{+} .
\end{array}\right.
$$

Case 2: Suppose that $X(t)$ in (4.1) is (ii)-differentiable, then we proceed as in Case 1 . Hence (4.3) can be rewritten in the sense of (ii)-differentiability as follows:

$$
\left\{\begin{array}{l}
\left(X^{+}(t)\right)^{\prime}=F^{-}(t)+\int_{t_{0}}^{t} \frac{k(t, s) X(s)}{\left(X^{-}(t)\right)^{\prime}=F^{+}(t)+\int_{t_{0}}^{t} \overline{k(t, s) X(s)} d s,} \\
X^{-}\left(t_{0}\right)=X_{0}^{-}, \\
X^{+}\left(t_{0}\right)=X_{0}^{+} .
\end{array}\right.
$$

Example 4.1 Let us consider the following IIDE:

$$
D_{H}^{g} X(t)=\int_{0}^{t} X(s) d s, \quad X(0)=X_{0}=[-1,1], t \in[0, \pi / 2] .
$$

Case 1. From (4.3), we get

$$
\left\{\begin{array}{l}
\left(X^{-}(t)\right)^{\prime}=\int_{0}^{t} X^{-}(s) d s \\
\left(X^{+}(t)\right)^{\prime}=\int_{0}^{t} X^{+}(s) d s, \\
X^{-}(0)=-1, \\
X^{+}(0)=1 .
\end{array}\right.
$$

By solving IDEs (4.6), we obtain $X_{1}(t)=\left[\frac{-\left(e^{t}+e^{-t}\right)}{2}, \frac{\left(e^{t}+e^{-t}\right)}{2}\right]$, and this solution is shown in Figure 1.

Figure 1 Solution of Example 4.1 in Case 1.

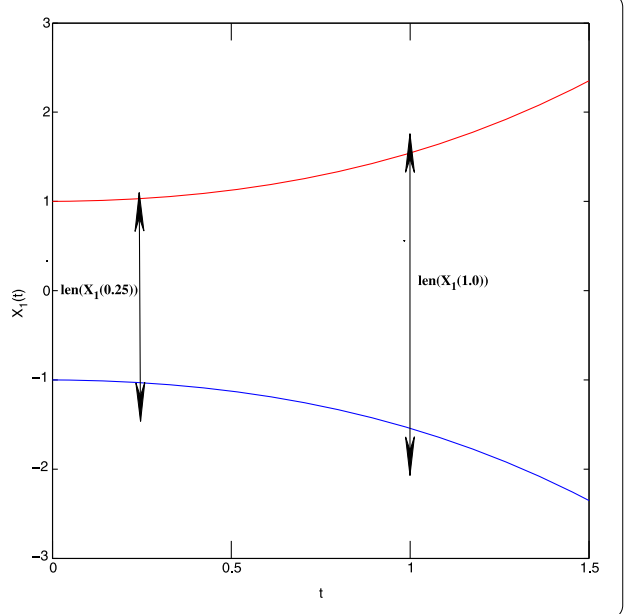


Case 2. From (4.4), we get

$$
\left\{\begin{array}{l}
\left(X^{+}(t)\right)^{\prime}=\int_{0}^{t} X^{-}(s) d s, \\
\left(X^{-}(t)\right)^{\prime}=\int_{0}^{t} X^{+}(s) d s, \\
X^{-}(0)=-1, \\
X^{+}(0)=1 .
\end{array}\right.
$$

By solving IDEs (4.7), we obtain $X_{2}(t)=[-\cos (t), \cos (t)]$, and this solution is shown in Figure 2 .

Example 4.2 Let us consider the following IIDE:

$$
D_{H}^{g} X(t)=[-2,2]+\int_{0}^{t} X(s) d s, \quad X(0)=X_{0}=[-2,2], t \in[0,0.5] .
$$

Case 1. We obtain $X_{1}(t)=\left[-2 e^{t}, 2 e^{t}\right]$, and this solution is shown in Figure 3 .

Case 2. We obtain $X_{2}(t)=[-2 \cos (t)+2 \sin (t), 2 \cos (t)-2 \sin (t)]$, and this solution is shown in Figure 4.

Figure 2 Solution of Example 4.1 in Case 2.

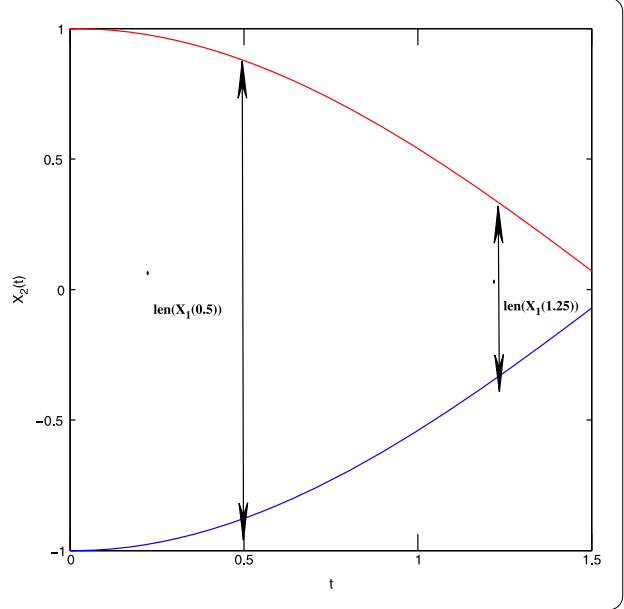

Figure 3 Solution of Example 4.2 in Case 1.

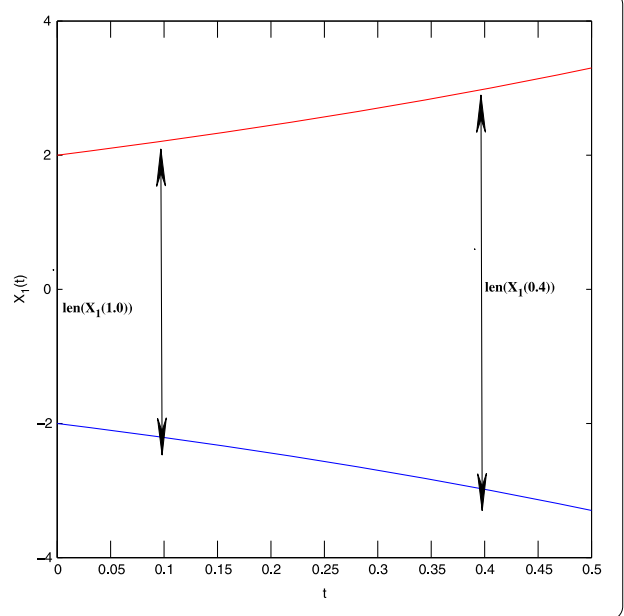




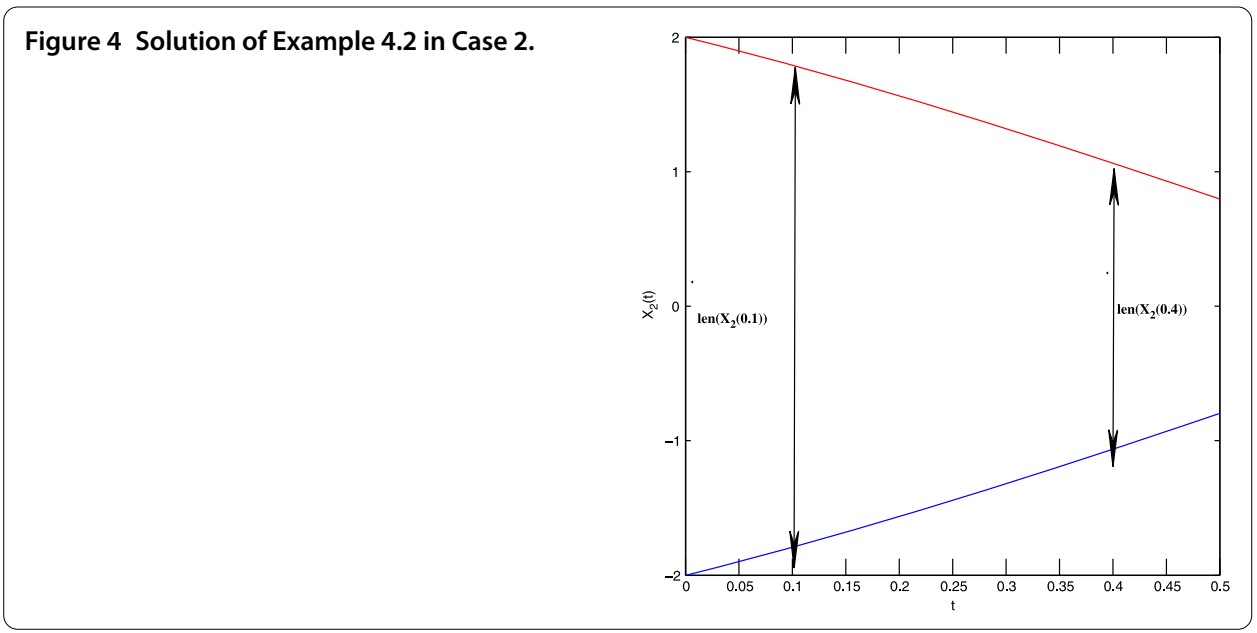

As we see in figures, the first type and second type Hukuhara differentiable intervalvalued solutions $X$ behave in various ways, i.e., one can say that len $\left(X_{1}(t)\right)$ in examples is nondecreasing in time (see Figures 1 and 3 ) and len $\left(X_{2}(t)\right)$ in examples is nonincreasing in time (see Figures 2 and 4).

\section{Conclusions and further work}

From Example 4.1 to Example 4.2, we notice that the solutions under the classical Hukuhara derivative ((i)-differentiable) have increasing length of their values. Indeed, we can see this in Figures 1 and 3. However, if we consider the second type Hukuhara derivative ((ii)-differentiable), the length of solutions changes. Under the second type Hukuhara derivative, differentiable solutions have nonincreasing length of its values (see Figures 2 and 4). In [17, 18], authors introduced and studied new generalized differentiability concepts for interval-valued functions. Our point is that the generalization of this concept can be of great help in the dynamic study of interval-valued differential equations and interval-valued integro-differential equations.

Competing interests

The authors declare that they have no competing interests.

Authors' contributions

All authors read and approved the final version of the manuscript.

\section{Author details}

${ }^{1}$ Faculty of Foundation Sciences, University of Technical Education, Ho Chi Minh City, Vietnam. ${ }^{2}$ Division of Applied Mathematics, Ton Duc Thang University, Nguyen Huu Tho Street, District 7, Ho Chi Minh City, Vietnam. ${ }^{3}$ Faculty of Mathematics and Computer Science, University of Science, VNU, Ho Chi Minh City, Vietnam.

\section{Acknowledgements}

The authors would like to express their gratitude to the anonymous referees for their helpful comments and suggestions, which have greatly improved the paper. The first named author would like to thank the University of Technical Education, Ho Chi Minh City, Vietnam.

Received: 10 January 2013 Accepted: 8 July 2013 Published: 22 July 2013

\section{References}

1. De Blasi, FS, lervolino, F: Equazioni differenziali con soluzioni a valore compatto convesso. Boll. Unione Mat. Ital. 4(2), 194-501 (1969)

2. Agarwal, RP, O'Regan, D: Existence for set differential equations via multivalued operator equations. Differ. Equ. Appl. 5, 1-5 (2007)

3. Agarwal, RP, O'Regan, D, Lakshmikantham, V: Viability theory and fuzzy differential equations. Fuzzy Sets Syst. 151(3), 563-580 (2005) 
4. Agarwal, RP, O'Regan, D, Lakshmikantham, V: A stacking theorem approach for fuzzy differential equations. Nonlinear Anal. TMA 55(3), 299-312 (2003)

5. Devi, JV: Generalized monotone iterative technique for set differential equations involving causal operators with memory. Int. J. Adv. Eng. Sci. Appl. Math. (2011). doi:10.1007/s12572-011-0031-1

6. Ngo, VH, Nguyen, DP: On maximal and minimal solutions for set-valued differential equations with feedback control. Abstr. Appl. Anal. (2012). doi:10.1155/2012/816218

7. Kaleva, O: Fuzzy differential equations. Fuzzy Sets Syst. 24, 301-317 (1987)

8. Lakshmikantham, V, Bhaskar, TG Devi, JV: Theory of Set Differential Equations in Metric Spaces. Cambridge Scientific Publisher, Cambridge (2006)

9. Puri, M, Ralescu, D: Differentials of fuzzy functions. J. Math. Anal. Appl. 91, 552-558 (1983)

10. Lakshmikantham, V, Mohapatra, R: Theory of Fuzzy Differential Equations and Inclusions. Taylor \& Francis, London (2003)

11. De Blasi, FS, Lakshmikantham, V, Bhaskar, TG: An existence theorem for set differential inclusions in a semilinear metric space. Control Cybern. 36(3), 571-582 (2007)

12. Song, $\mathrm{S}, \mathrm{Wu}, \mathrm{C}$ : Existence and uniqueness of solutions to Cauchy problem of fuzzy differential equations. Fuzzy Sets Syst. 110, 55-67 (2000)

13. Bhaskar, TG, Lakshmikantham, V: Set differential equations and flow invariance. J. Appl. Anal. 82(2), 357-368 (2003)

14. Hale, JK: Theory of Functional Differential Equations. Springer, New York (1977)

15. Kolmanovskii, VB, Myshkis, A: Applied Theory of Functional Differential Equations. Kluwer Academic, Dordrecht (1992)

16. Park, JY, Jeong, JU: On existence and uniqueness of solutions of fuzzy integro-differential equations. Indian J. Pure Appl. Math. 34(10), 1503-1512 (2003)

17. Stefanini, L, Bede, B: Generalized Hukuhara differentiability of interval-valued functions and interval differential equations. Nonlinear Anal. TMA 71, 1311-1328 (2009). doi:10.1016/j.na.2008.12.005

18. Chalco-Cano, Y, Rufián-Lizana, A, Román-Flores, H, Jiménez-Gamero, MD: Calculus for interval-valued functions using generalized Hukuhara derivative and applications. Fuzzy Sets Syst. (2012). doi:10.1016/j.fss.2012.12.004

19. Malinowski, MT: Interval Cauchy problem with a second type Hukuhara derivative. Inf. Sci. 213, 94-105 (2012). doi:10.1016/j.ins.2012.05.022

20. Malinowski, MT: Interval differential equations with a second type Hukuhara derivative. Appl. Math. Lett. 24 2118-2123 (2011)

21. Allahviranloo, T, Hajighasemi, S, Khezerloo, M, Khorasany, M, Salahshour, S: Existence and uniqueness of solutions of fuzzy Volterra integro-differential equations. In: IPMU. CCIS, vol. 81, pp. 491-500 (2010)

22. Allahviranloo, T, Amirteimoori, A, Khezerloo, M, Khezerloo, S: A new method for solving fuzzy Volterra integro-differential equations. Aust. J. Basic Appl. Sci. 5(4), 154-164 (2011)

23. Allahviranloo, T, Salahshour, S, Abbasbandy, S: Solving fuzzy fractional differential equations by fuzzy Laplace transforms. Commun. Nonlinear Sci. Numer. Simul. 17(3), 1372-1381 (2012). doi:10.1016/j.cnsns.2011.07.005

24. Allahviranloo, T, Abbasbandy, S, Sedaghatfar, O, Darabi, P: A new method for solving fuzzy integro-differential equation under generalized differentiability. Neural Comput. Appl. 21(1), 191-196 (2012). doi:10.1007/s00521-011-0759-3

25. Allahviranloo, T, Ghanbari, M, Haghi, E, Hosseinzadeh, A, Nouraei, R: A note on 'Fuzzy linear systems'. Fuzzy Sets Syst. $177,87-92(2011)$

26. Alikhani, R, Bahrami, F, Jabbari, A: Existence of global solutions to nonlinear fuzzy Volterra integro-differential equations. Nonlinear Anal. TMA 75(4), 1810-1821 (2012)

27. Bede, B, Gal, SG: Generalizations of the differentiability of fuzzy-number-valued functions with applications to fuzzy differential equations. Fuzzy Sets Syst. 151, 581-599 (2005)

28. Bede, B, Rudas, IJ, Bencsik, AL: First order linear fuzzy differential equations under generalized differentiability. Inf. Sci. $177,1648-1662(2007)$

29. Bede, B, Stefanini, L: Generalized differentiability of fuzzy-valued functions. Fuzzy Sets Syst. (2012). doi:10.1016/j.fss.2012.10.003

30. Bede, B: A note on 'Two-point boundary value problems associated with non-linear fuzzy differential equations'. Fuzzy Sets Syst. 157(7), 986-989 (2006)

31. Chalco-Cano, Y, Román-Flores, H: On new solutions of fuzzy differential equations. Chaos Solitons Fractals 38, $112-119(2008)$

32. Lakshmikantham, $\mathrm{V}$, Tolstonogov, AA: Existence and interrelation between set and fuzzy differential equations. Nonlinear Anal. TMA 55(3), 255-268 (2003)

33. Stefanini, L, Sorini, L, Guerra, ML: Parametric representation of fuzzy numbers and application to fuzzy calculus. Fuzzy Sets Syst. 157, 2423-2455 (2006)

34. Nguyen, DP, Ngo, VH, Ho, V: On comparisons of set solutions for fuzzy control integro-differential systems. J. Adv. Res. Appl. Math. 4(1), 84-101 (2012). doi:10.5373/jaram

35. Zhang, D, Feng, W, Zhao, Y, Qiu, J: Global existence of solutions for fuzzy second-order differential equations under generalized H-differentiability. Comput. Math. Appl. 60, 1548-1556 (2010)

36. Malinowski, MT: Random fuzzy differential equations under generalized Lipschitz condition. Nonlinear Anal., Real World Appl. 13(2), 860-881 (2012)

37. Malinowski, MT: Existence theorems for solutions to random fuzzy differential equations. Nonlinear Anal., Theory Methods Appl. 73(6), 1515-1532 (2010)

38. Malinowski, MT: On random fuzzy differential equations. Fuzzy Sets Syst. 160(21), 3152-3165 (2009)

39. Mizukoshi, MT, Barros, LC, Chalco-Cano, Y, Román-Flores, H, Bassanezi, RC: Fuzzy differential equations and the extension principle. Inf. Sci. 177, 3627-3635 (2007)

40. Malinowski, MT: On set differential equations in Banach spaces - a second type Hukuhara differentiability approach. Appl. Math. Comput. 219(1), 289-305 (2012)

41. Malinowski, MT: Second type Hukuhara differentiable solutions to the delay set-valued differential equations. Appl. Math. Comput. 218(1), 9427-9437 (2012)

42. Ngo, VH: Interval-valued integro-differential equations under generalized $\mathrm{H}$-differentiability (to appear) 
doi:10.1186/1687-1847-2013-217

Cite this article as: Truong et al.: Global existence of solutions for interval-valued integro-differential equations under generalized H-differentiability. Advances in Difference Equations 2013 2013:217.

Submit your manuscript to a SpringerOpen ${ }^{\circ}$ journal and benefit from:

- Convenient online submission

- Rigorous peer review

- Immediate publication on acceptance

- Open access: articles freely available online

- High visibility within the field

- Retaining the copyright to your article

Submit your next manuscript at $\gg$ springeropen.com 